\title{
Structural characterization of adamantane-resistant mutants of the influenza M2 proton channel
}

Jessica L. Thomaston¹, William F. DeGrado ${ }^{1}$

1 Department of Pharmaceutical Chemistry, University of California, San Francisco

The M2 protein is a homotetrameric proton channel found in the influenza A virus. It is the target of two of the four FDA-approved anti-influenza drugs: amantadine and rimantadine. However, in the past decade, viral resistance has emerged in the majority of currently circulating strains of influenza. The most prevalent drugresistant M2 mutants are S31N and V27A [1]. Here we report X-ray crystal structures of both of these mutants. A newly solved $2.1 \AA$ structure of the S31N mutant contains two conformations of the channel (Inward open $_{\text {and }}$ Inward closed $_{\text {) }}$ in the asymmetric unit of a novel crystal form, allowing us to observe the Inward closed $_{\text {in }}$ conformation of the S31N mutant for the first time. In the Inward open $_{\text {conformation, }}$ Asn 31 faces the center of the channel pore and sterically occludes the binding site of the adamantane drugs, as previously observed [2]. In the Inward closed $_{\text {conformation, }}$ Asn31 faces away from the channel pore and instead forms hydrogen bond interactions with backbone carbonyls, resulting in a wider channel pore at the adamantane binding site. We have also characterized the V27A mutant bound to a spiroadamantane inhibitor in a $2.5 \AA$ structure. The mutation of Val to Ala removes hydrophobic contacts that previously stabilized binding of the adamantane drugs, and the spiroadamantane inhibitor is observed to bind to this larger pocket. This work provides a structural explanation for adamantane resistance in the M2 channel that will be useful for the design of new compounds targeting these drugresistant mutants.

[1] Dong et al., PLoS One 2015.

[2] Thomaston et al., Protein Science 2016. 